\title{
Percepções dos alunos sobre a mudança do curso presencial para o remoto durante a pandemia de COVID-19: um estudo de caso do ensino de Química Geral
}

\author{
Students' perceptions on the change from in-person to remote course along COVID-19 outbreak: a \\ case study for General Chemistry \\ Percepciones de los estudiantes sobre el cambio de cursos presenciales a cursos remotos durante la \\ pandemia CONVID-19: un estudio de caso en la enseñanza de Química General
}

\author{
Luzia Peres Novaki \\ ORCID: https://orcid.org/0000-0002-1518-0724 \\ Universidade Federal do ABC, Brasil \\ E-mail: lpnovaki@yahoo.com.br \\ Juliana Marchi \\ ORCID: https://orcid.org/0000-0002-0663-0463 \\ Universidade Federal do ABC, Brasil \\ E-mail: juliana.marchi@ufabc.edu.br \\ Alvaro Takeo Omori \\ ORCID: https://orcid.org/0000-0002-0115-3485 \\ Universidade Federal do ABC, Brasil \\ E-mail: alvaro.omori@ufabc.edu.br \\ Solange Wagner Locatelli \\ ORCID: https://orcid.org/0000-0002-7639-6772 \\ Universidade Federal do ABC, Brasil \\ E-mail: solange.locatelli@ufabc.edu.br
}

\begin{abstract}
Resumo
O curso de Química Geral compõe a grade curricular obrigatória a ser cursada pelos graduandos, para obtenção do diploma de Bacharel em Ciência e Tecnologia na Universidade Federal do ABC. Durante o curso, são previstas aulas presenciais, porém devido ao cenário de pandemia do COVID-19, em 2020 o curso foi inicialmente ministrado presencialmente, e adaptado posteriormente para o modo remoto. Após o término das aulas, os alunos relataram suas percepções a partir do preenchimento de um questionário, com questões relacionadas à acessibilidade do formato remoto, percepções das aulas teóricas e de laboratórios, aprendizagem, autoavaliação e visão geral do curso, enfocando a mudança ocorrida quanto à modalidade de oferta. Quanto às aulas teóricas, os resultados indicaram que as percepções não parecem ter sido alteradas pela mudança do modo presencial para o remoto. Por outro lado, de acordo com suas percepções, laboratórios remotos não possibilitaram o desenvolvimento de habilidades cognitivas essenciais e práticas. Apesar dessas limitações, os resultados gerais demonstraram uma percepção positiva do curso, permitindo a conclusão da disciplina de forma satisfatória. Esses resultados podem se constituir de subsídios e precauções a serem considerados para futuros planejamentos de disciplinas de forma híbrida, sobretudo com relação às limitações inerentes ao modo remoto.
\end{abstract}

Palavras-chave: Química geral; Experimentação; Modo remoto; Percepção discente; Contexto pandêmico.

\begin{abstract}
The course of General Chemistry is part of the mandatory curriculum to be taken by undergraduates to obtain a Bachelor's degree in Science and Technology at the Federal University of ABC. During the course, face-to-face classes are planned, but due to the COVID-19 pandemic scenario, in 2020 the course was initially taught in person, and later adapted to remote mode. After the end of the classes, students reported their perceptions by filling out a questionnaire related to accessibility to remote mode, perceptions of theoretical classes and laboratories, learning, self-assessment and an overview of the course, focusing on the change that occurred when to the offer modality. As for the theoretical classes, the results indicated that perceptions do not seem to have been altered by the change from face-to-face to remote mode. On the other hand, according to their perceptions, remote laboratories did not allow the development of essential cognitive and practical skills. Despite these limitations, the overall results showed a positive perception of the course, allowing the course to be completed satisfactorily. These results can constitute subsidies and precautions to be considered for future planning of disciplines in a hybrid way, especially regarding the limitations inherent to the remote mode.
\end{abstract}

Keywords: General chemistry; Experimentation; Remote mode; Students' perceptions; Pandemic context. 


\begin{abstract}
Resumen
El curso de Química General es parte del plan de estudios obligatorio que deben tomar los estudiantes universitarios para obtener el título de Licenciado en Ciencia y Tecnología en la Universidad Federal de ABC. Durante el curso se planifican clases presenciales, pero debido al escenario de la pandemia COVID-19, en 2020 el curso se impartió inicialmente de manera presencial, y luego se adaptó a modo remoto. Una vez finalizadas las clases, los estudiantes informaron sus percepciones mediante la cumplimentación de un cuestionario, con preguntas relacionadas con la accesibilidad del formato remoto, percepciones de las clases teóricas y laboratorios, aprendizaje, autoevaluación y una visión general del curso, enfocándose en el cambio que se produjo cuando a la modalidad de oferta. En cuanto a las clases teóricas, los resultados indicaron que las percepciones no parecen haber sido alteradas por el cambio de modo presencial a remoto. Por otro lado, según sus percepciones, los laboratorios remotos no permitían el desarrollo de habilidades cognitivas esenciales y prácticas. A pesar de estas limitaciones, los resultados generales mostraron una percepción positiva del curso, lo que permitió completarlo satisfactoriamente. Estos resultados pueden constituir subsidios y precauciones a considerar para la planificación futura de disciplinas de manera híbrida, especialmente en lo que respecta a las limitaciones inherentes al modo remoto.
\end{abstract}

Palabras clave: Química general; Experimentación; Modo remoto; Percepción del alumno; Contexto pandémico.

\title{
1. Introdução
}

Em todo o mundo, educadores e sistemas educacionais têm enfrentado uma mudança drástica e global em sua forma de ensino, devido ao surto de SARS-CoV-2 (Coronavírus, 2021; McCarthy, 2020).

No Brasil, assim como em muitos outros países, o fechamento de escolas e universidades, em meados de março de 2020, causou uma súbita interrupção do processo de ensino e aprendizagem presencial (Croda et al., 2020). Os alunos, professores e funcionários das universidades foram obrigados a cumprir um regime de quarentena (Brasil, 2021). Diante desse cenário, as atividades tiveram que ser adaptadas repentinamente para uma perspectiva remota (Brasil, 2021a; Tam \& El-Azar, 2020) que, segundo Guerra Júnior et al. (2021) se revelou como uma forma de dar espaço para outras metodologias a serem inseridas no contexto educacional, antes pouco utilizadas, por variadas razões.

$\mathrm{O}$ ano acadêmico brasileiro geralmente começa em fevereiro e tem uma pausa de inverno em julho. Na Universidade Federal do ABC (UFABC), localizada no cinturão industrial da cidade de São Paulo, o ano letivo se divide em três quadrimestres com 12 semanas de aulas cada. A UFABC tem uma proposta de ensino diferenciada e destaca a importância de uma educação inovadora que prioriza as habilidades de inserção social dos alunos em um sentido mais amplo. Leva em consideração o dinamismo da ciência, bem como oferece uma matriz interdisciplinar, proporcionando aos novos profissionais alguns conhecimentos mais abrangentes, tornando-os capazes de transitarem facilmente nas diversas áreas científicas e tecnológicas. Para essa proposta pedagógica, os alunos aderem inicialmente ao curso de Bacharelado em Ciência e Tecnologia (BCT), onde nos dois primeiros anos cursam disciplinas que abrangem as ciências humanas, exatas e biológicas. Em seguida, um curso específico pode ser escolhido, dependendo de sua afinidade, entre os quais destacam-se engenharia ou química. Esta visão mais ampla permite ao aluno aprender sobre os principais conceitos básicos subjacentes em diversas áreas do conhecimento, além de desenvolver suas habilidades em laboratório.

Neste contexto, o curso de Química Geral é ofertado aos alunos de graduação durante o primeiro ano, como requisito para a obtenção do grau de BCT. Intitulado como "Transformações Químicas" (doravante denominado TransQuim), este curso quadrimestral envolve tópicos básicos em química geral, como interações intermoleculares, estequiometria, termoquímica, cinética, equilíbrio e teoria ácido-base.

Quando as atividades presenciais da universidade foram interrompidas, devido ao surto de SARS-CoV-2, aproximadamente metade do curso planejado já havia sido oferecido presencialmente. Em um cenário incerto à época, logo após a suspensão das atividades presenciais, a UFABC adotou um momento de transição denominado Ensino Continuado Emergencial (ECE), no qual todas as atividades foram adaptadas levando-se em consideração a modalidade exclusivamente remota (UFABC, 2020). Portanto, o processo de ensino-aprendizagem pôde continuar após um curto período de interrupção, 
em que os alunos puderam finalizar o curso realizando atividades remotas. A Figura 1 mostra os marcos epidêmicos no Brasil e o período de ECE na UFABC.

Figura 1. Linha do tempo do cenário pandêmico que culminou na modalidade ECE na UFABC e no curso de TransQuim em modo remoto.

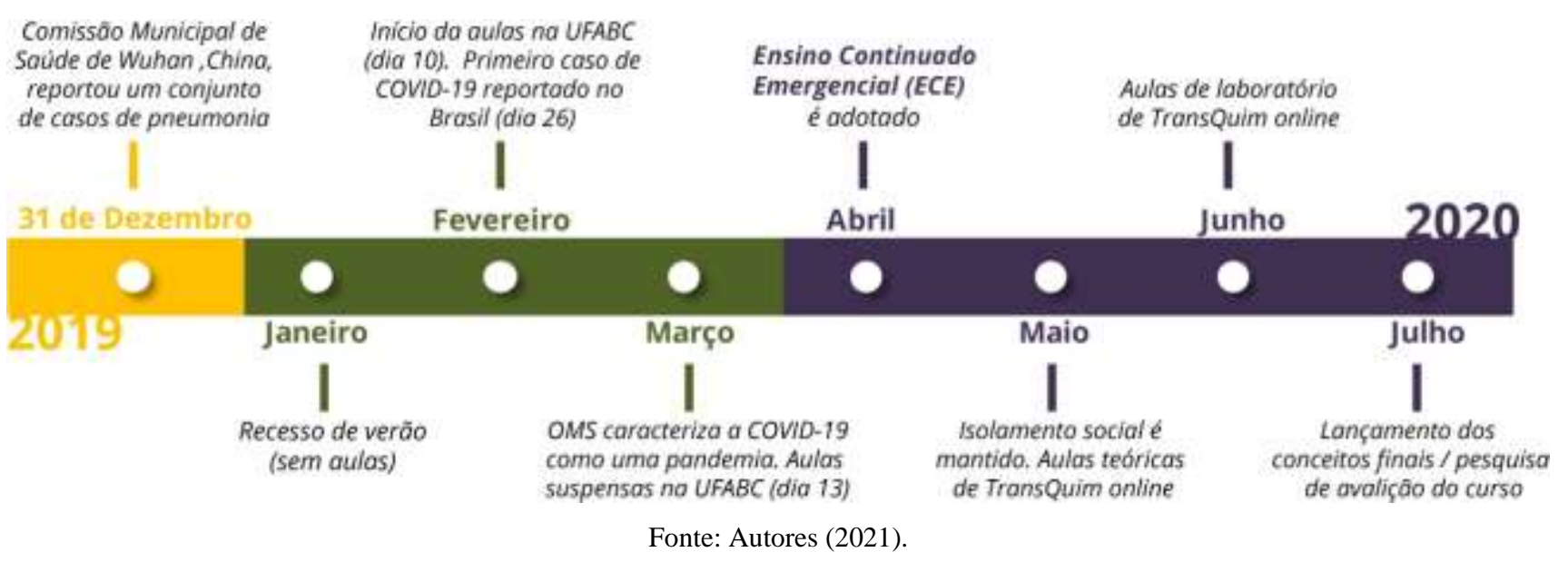

O ensino remoto não era uma realidade na UFABC: a maioria dos professores não adotava plataformas de ensino e aplicativos didáticos para o ensino a distância, os alunos não estavam preparados para participar de atividades em ambiente remoto e as experiências de laboratório eram voltadas, exclusivamente, para atividades práticas presenciais. Com o plano de ECE proposto pela UFABC, os professores foram desafiados a se comunicar, ensinar conceitos químicos, motivar os alunos e avaliar o aprendizado por meio de atividades remotas. Os alunos, por sua vez, tiveram que se adaptar a um processo de aprendizagem virtual, superando desafios que incluem conexão com a internet, falta de interação com seus colegas e desenvolvimento de autonomia de aprendizagem.

Esses problemas não foram exclusivos da UFABC. A maioria das comunidades universitárias em todo o mundo enfrentaram desafios semelhantes. Membros de muitas instituições acadêmicas, incluindo alunos, professores e assistentes, relataram seus desafios e sucessos quanto à adaptação do curso presencial para o modo online (Babinčáková \& Bernard, 2020; Dickson-Karn, 2020; Dietrich et al., 2020; Emenike et al., 2020; George-Williams et al., 2020; Huang, 2020; Koirala et al., 2020; Kolack et al., 2020; Ranga, 2020; Rodríguez-Rodríguez et al., 2020; Soares et al., 2020; Talanquer et al., 2020; Tan et al., 2020; Villanueva et al., 2020; Wenzel, 2020).

Durante o surto de COVID-19, muitos cursos de química foram alterados para oferecer aulas experimentais online, seja por demonstração em vídeo do próprio professor ou por vídeo disponibilizado na internet, com aulas síncronas ou assíncronas (George-Williams et al., 2020; Howitz et al., 2020; Ranga, 2020; Villanueva et al., 2020), ou substituição das atividades de laboratório pela análise dos dados obtidos por grupos anteriores e fornecidos aos alunos (Dietrich et al., 2020). Outras propostas incluíram a aprendizagem colaborativa em grupo, usando métodos de ensino remoto síncronos (Wenzel, 2020), bem como experimentos práticos de química realizados pelos alunos em suas residências (Selco, 2020).

Neste contexto, as percepções dos alunos sobre a mudança de cursos presenciais para remotos são de suma importância para identificar itens relevantes que podem afetar este processo (Wart et al., 2020). Por exemplo, os alunos relataram que as aulas teóricas mudaram pouco em relação a uma aula tradicional, enquanto os laboratórios mudaram drasticamente, passando de experiências em que o aluno tem uma postura ativa, com tomada de decisões, para uma postura passiva, de observação (Jeffery \& Bauer, 2020). Além disso, alguns alunos relataram uma avaliação não positiva na aprendizagem online devido à interrupção da internet, a ausência de um laboratório de química virtual, estresse e falta de 
interatividade (Blizak et al., 2020), muito embora outros grupos de pesquisa tenham relatado uma preferência dos alunos da instrução síncrona ao modo assíncrona (Rodríguez-Rodríguez et al., 2020; Wenzel, 2020). No entanto, os alunos pareciam exibir desempenho semelhante tanto no curso presencial quanto no remoto (Huang, 2020; Selco, 2020), mas se sentiam frustrados e menos engajados ao contexto universitário devido à limitada interação professor-aluno (Huang, 2020). Também foram relatadas algumas dificuldades quanto à elaboração de relatórios de laboratório, uma vez que os alunos não realizaram os experimentos por conta própria (Dickson-Karn, 2020).

A hipótese deste trabalho é que o processo de aprendizagem de nossos alunos pode ter sido comprometido pelas condições atípicas ao longo da mudança de modelos presenciais para remotos. No entanto, os alunos podem ter percepções diferentes sobre sua aprendizagem que devem ser consideradas. Assim, o interesse nesta pesquisa foi investigar a percepção dos alunos sobre sua aprendizagem, interesse e desempenho no curso de TransQuim, durante o ano de 2020, no contexto pandêmico.

Para isso, um questionário foi elaborado para explorar suas percepções, particularmente no que diz respeito a i) acessibilidade e aceitação do ECE; ii) aulas teóricas e de laboratório, enfocando a importância da articulação entre elas para a compreensão e aprendizagem dos conceitos químicos em cada tema, abordando facilidades e dificuldades na mudança do modo presencial para o remoto; iii) aprendizagem por meio de aulas de laboratório; iv) autoavaliação de interesse e desenvolvimento de habilidades cognitivas e v) visão geral do curso, considerando-se tanto as aulas teóricas como as de laboratório.

\section{Metodologia}

\subsection{Estrutura da disciplina de TransQuim presencial}

No âmbito da graduação do BCT, o curso de TransQuim é o primeiro a ter um laboratório voltado para a química. É usualmente planejado para ser oferecido em um período de 12 semanas, sendo que cada semana contempla três horas de aulas teóricas e duas horas dedicadas às aulas de laboratório. As aulas teóricas são ministradas com base no programa de Química Geral, com o apoio de monitores, visando à resolução de exercícios e auxílio no esclarecimento de dúvidas. Em geral, os alunos são avaliados por dois exames.

As aulas de laboratório são propostas para se articular com os conceitos já abordados nas aulas teóricas, tendo como objetivo a compreensão e aprendizagem dos conceitos químicos desenvolvidos, bem como proporcionar um contato com os conteúdos procedimentais como, por exemplo, manusear vidrarias e reagentes, visando também o desenvolvimento de uma atividade na perspectiva investigativa, que de acordo com Zômpero e Laburú (2011) contemplam alguns elementos importantes como o levantamento e teste de hipóteses, coleta e interpretação dos dados obtidos, com a construção dos conceitos feita de forma coletiva entre todos. Ao todo, seis experimentos foram planejados ao longo de 2020, como pode ser observado na Tabela 1.

Tabela 1. Descrição sucinta dos experimentos de laboratório realizados na disciplina de TransQuim, ofertada em 2020.

\begin{tabular}{llll}
\hline Modo & Exp & Titulo & Período \\
\hline \multirow{2}{*}{ Presencial } & 1 & Determinação da concentração de sacarose em refrigerantes & Fevereiro \\
\cline { 2 - 4 } & 2 & Interações Intermoleculares & Fevereiro \\
\hline \multirow{2}{*}{ Remoto } & 4 & Determinação do teor de bicarbonato em comprimidos efervescentes & Março \\
\hline & 5 & Entalpia de decomposição do peróxido de hidrogênio & Junho \\
\hline & 6 & Cinética química & Junho \\
\hline
\end{tabular}

Fonte: Autores (2021). 
A cada aula prática, os alunos são incentivados a resolver problemas práticos relacionados com os temas específicos abordados, com base em um roteiro de laboratório fornecido pelo professor, numa perspectiva investigativa de acordo com cada temática. Os alunos são desafiados a encontrar, em grupos (de 4 a 5 pessoas), uma solução para o problema prático abordado, usando os equipamentos, vidrarias e técnicas experimentais disponíveis no laboratório, e preparam um relatório a ser entregue imediatamente após cada aula. Além disso, a avaliação das atividades práticas inclui o conteúdo trabalhado no relatório. Em geral, após três sessões de laboratório, é prevista uma aula de discussão, para análise crítica dos resultados obtidos pelos grupos de trabalho com base nos resultados esperados. Um exame individual é aplicado, incluindo perguntas abertas para se avaliar a aprendizagem no laboratório. A nota final atribuída ao aluno no curso de TransQuim é composta por avaliações teóricas e práticas.

\subsection{Estrutura da disciplina de TransQuim ofertada remotamente}

Em função da pandemia de COVID-19, algumas adaptações na estrutura do curso de TransQuim foram feitas para que ficassem de acordo com o plano de ensino adotado anteriormente. No início, conforme o planejamento do ECE, apenas as aulas teóricas da disciplina foram oferecidas remotamente. Entre os meses de março e abril de 2020, não se tinha ideia da extensão da quarentena, portanto, esperávamos que as aulas de laboratório pudessem ser realizadas presencialmente em um futuro próximo. A adesão ao plano ECE não foi obrigatória para os alunos, uma vez que a diversidade socioeconômica e os problemas de acessibilidade no ensino remoto poderiam interferir no processo. A Figura 2 apresenta os principais eventos relacionados ao curso de TransQuim ofertado parcialmente em 2020.

Figura 2. Cronograma dos eventos do curso de TransQuim ofertado parcialmente, durante o surto de COVID-19 em 2020.

Aulas teóricas presenciais
Fevereiro

Fonte: Autores (2021).

As aulas teóricas remotas (Figura 2) foram realizadas semanalmente de modo síncrono (2 horas), utilizando as ferramentas de streaming de vídeo GoogleMeet e de modo assíncrono (1 hora), principalmente pela disponibilização de vídeos gravados pelo professor abordando a resolução dos exercícios. Aulas remotas adicionais foram realizadas de modo síncrono para revisão de conceitos e resolução de problemas. Todas as aulas remotas síncronas e assíncronas ficaram disponíveis aos alunos no site do YouTube (TQ, 2020). No final do curso, compreendendo o conjunto das aulas teóricas, foi aplicado um exame on-line, com questões dissertativas, abrangendo todos os conceitos ministrados ao longo do quadrimestre letivo.

Após um período de 7 semanas de curso teórico ofertado remotamente, o cenário pandêmico se intensificou e não foi permitida a retomada das aulas presenciais de laboratório. Assim, aulas de laboratório remoto foram oferecidas aos alunos para finalização do curso. Com a suspensão das atividades presenciais na Universidade, os professores não puderam produzir seus próprios materiais sobre os experimentos usando a infraestrutura dos laboratórios da UFABC. Não obstante, foi mantido o roteiro experimental, com alguma adequação. Os professores realizaram e disponibilizaram aos alunos vídeo-slides comentados sobre a prática experimental proposta, com ênfase no procedimento experimental e nos resultados esperados. O 
conteúdo de cada experimento foi disponibilizado de forma assíncrona numa plataforma do Ambiente Virtual de Aprendizagem. Semanalmente, os alunos apresentavam um relatório individual, contendo respostas às questões e achados dos experimentos. Os professores estiveram disponíveis todas as semanas por meio de encontros síncronos na plataforma GoogleMeet para discutir os resultados, esclarecer dúvidas sobre as atividades propostas e promover um contato mais efetivo com os alunos. As dúvidas também foram resolvidas diretamente por e-mail ou encontros síncronos sob demanda.

\subsection{Questionário para avaliar as percepções dos alunos}

Um questionário foi formulado para avaliar as percepções durante o curso de TransQuim. Este questionário abordou os tópicos mostrados na Tabela 2, enfocando na adaptação do curso do modo presencial para o modo remoto.

Tabela 2. Tópicos do questionário sobre a percepção dos alunos.

\begin{tabular}{ll}
\hline Tópico & Detalhes \\
\hline $\begin{array}{l}\text { Acessibilidade e adaptações } \\
\text { no modo ECE }\end{array}$ & $\begin{array}{l}\text { Razões para o acesso ao plano ECE e possíveis melhorias de internet e espaços domiciliares para o } \\
\text { estudo on-line. }\end{array}$ \\
\hline $\begin{array}{l}\text { Estrutura das aulas teóricas e } \\
\text { de laboratório }\end{array}$ & $\begin{array}{l}\text { A importância dos experimentos para articulação com os conceitos teóricos; a importância das aulas de } \\
\text { teoria para articulação com os laboratórios ofertados remotamente; facilidades e dificuldades na } \\
\text { mudança do modo presencial para o remoto com relação às aulas de teoria e laboratório. }\end{array}$ \\
\hline $\begin{array}{l}\text { Aprendizado durante as aulas } \\
\text { de laboratório }\end{array}$ & $\begin{array}{l}\text { Entendimento de conteúdos químicos baseados nas aulas de laboratório, ofertadas de modo presencial ou } \\
\text { remoto. }\end{array}$ \\
\hline Autoavaliação & $\begin{array}{l}\text { Interesse em cada experimento; desenvolvimento de habilidades cognitivas; atitude, postura e } \\
\text { gerenciamento das atividades. }\end{array}$ \\
\hline Curso em geral & $\begin{array}{l}\text { Autoavaliação da dedicação e desempenho durante o curso ofertado presencialmente e de modo remoto; } \\
\text { Avaliação geral do curso. }\end{array}$ \\
\hline
\end{tabular}

Fonte: Autores (2021).

As questões foram formuladas de maneira a identificar as percepções em um formato de resposta em escala Likert de 5 pontos, considerando de 1 (ruim) a 5 (muito bom) e com questões abertas. A participação dos alunos foi voluntária ( $\mathrm{n}=42$ ). Os questionários foram disponibilizados na plataforma GoogleForms para serem respondidos até duas semanas após o término das aulas (Figura 2). Ressalta-se que a presente pesquisa foi registrada no Comitê de Ética do Brasil, endereço eletrônico da Plataforma Brasil, sob o código CAAE n 46902221.8.00005594, sendo aprovada pelo Comitê de Pesquisas da Universidade Federal do ABC.

\section{Resultados e Discussão}

A adaptação do curso de TransQuim pode ser entendida como parte do plano CIPPr (Contexto, Imput, Processo, Produto) (Hodges et al., 2020; Stufflebeam \& Zhang, 2017). O contexto, (C), definido como mudança de atividades devido à pandemia, gerou a necessidade de uma alteração no plano de atividades, originalmente planejado para ser executado de forma presencial. As atividades remotas culminaram no programa ECE (I, do Imput). O processo, (P), pode ser entendido como o oferecimento de aulas de teoria e laboratório no modo remoto, com o constante monitoramento do processo de aprendizado dos alunos baseado na entrega de relatórios semanais, encontros síncronos e contato por e-mail. Após o termino das atividades, um questionário avaliativo (produto, Pr) foi elaborado para entender a percepção dos alunos sobre o curso, com o foco na mudança do modelo de aulas presencial para o remoto.

Questionários são considerados ferramentas úteis para avaliar a percepção dos alunos sobre o progresso do aprendizado e estrutura do curso. Ademais, são eficientes em se compreender os processos de aprendizagem vivenciados (Dickson-Karn, 2020; Hensen et al., 2020; Kolil et al., 2020; Winkelmann et al., 2017). 
Neste trabalho, houve uma grande preocupação em avaliar diferenças entre as experiências vividas presencialmente e de forma remota durante o curso de TransQuim, de acordo com as perspectivas dos alunos. Os resultados da pesquisa foram, portanto, agrupados em cinco principais tópicos: acessibilidade e regras do jogo no modo ECE; estrutura das aulas teóricas e de laboratório; aprendizado; autoavaliação; perspectiva geral do curso.

A seguir, é apresentada uma análise crítica de cada tópico do questionário.

\subsection{Acessibilidade e adaptações no modo ECE}

Para dar continuidade ao curso TransQuim à distância, adaptações se fizeram necessárias. Estes alunos que continuaram o curso no modelo ECE tiveram acesso à internet e poucos foram os relatos de conexão regular ou ruim. Em relação ao hardware, todas as respostas foram positivas quanto à qualidade dos equipamentos (celular, computador, laptop) utilizados por eles.

No entanto, durante o ECE, muitos alunos avaliaram o espaço em casa para realizar as atividades como de qualidade regular. Poucos relataram que o espaço era muito bom, e muitos tiveram que fazer algumas adaptações quanto à acessibilidade para a realização da disciplina, como explicitado nos seguintes relatos:

"Tive que trocar os móveis e ajustar os cômodos da casa mais distantes dos cômodos principais, buscando mais silêncio e conforto para acompanhar as aulas, bem como a aquisição de aparelhos eletrônicos mais modernos para maior praticidade;

"Tive que comprar um fone de ouvido com microfone e contratar serviços de internet mais potentes".

Quando questionados sobre o motivo do ingresso no modo ECE para frequentar as aulas de laboratório remoto, a maioria dos alunos relatou preocupação em terminar os cursos, não atrasar a formatura e continuar os estudos naquele momento. Alguns relatos podem ser observados na íntegra, reproduzidos a seguir.

"Terminar o curso em breve e não ter que comparecer aos laboratórios em conjunto com outras disciplinas que não estejam relacionadas com o retorno das aulas";

"Acredito que pude aprender da mesma forma que presencialmente (obviamente não a parte prática, porém os conceitos); além disso, não gostaria de estender a disciplina além do período de quatro meses previsto";

"Entrei porque não queria adiar a formatura, tendo que fazer depois";

\subsection{Percepções dos estudantes sobre aulas teóricas e de laboratório}

A estrutura geral do curso de TransQuim compreende aulas teóricas e de laboratório. Durante o modo presencial, os experimentos foram realizados simultaneamente com as aulas teóricas (experimentos 1 a 3). Já considerando as aulas ministradas no modo remoto, os experimentos (de 4 a 6) foram realizados após o final do período das aulas teóricas, conforme Figura 2.

Neste contexto, foram avaliadas as percepções sobre quanto os conteúdos teórico e prático foram complementares para obter uma compreensão geral do curso de TransQuim (Figura 3). 
Figura 3. A) Avaliação da importância das aulas laboratoriais para a compreensão dos temas abordados nas aulas teóricas. B) Avaliação da importância dos assuntos abordados na teoria para a realização dos experimentos.

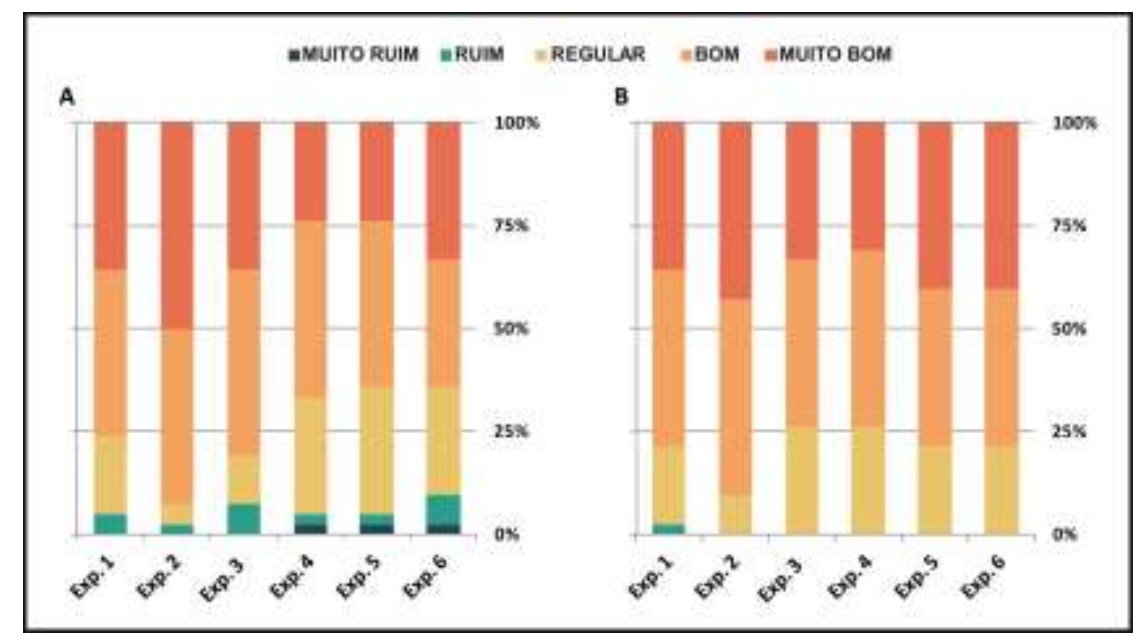

Fonte: Autores (2021).

No que diz respeito aos laboratórios presenciais e remotos, cerca de $80 \%$ e $60 \%$ dos alunos, respetivamente, apontaram como positivos a importância das aulas laboratoriais para a compreensão dos temas abordados nas aulas teóricas, considerando as escalas boas ou muito boas (Figura 3A).

Parece que os alunos tiveram mais dificuldade em entender os conceitos abordados quando os laboratórios foram realizados no modo remoto, uma vez que tais experimentos foram realizados muito depois das aulas teóricas, ficando desconectados dos conceitos discutidos nas aulas teóricas. Essas percepções podem ser explicadas pela ausência de interação direta com um professor, que poderia ter proporcionado uma comunicação mais adequada relacionada aos procedimentos experimentais nas aulas de laboratório, bem como a ausência de experiências de aprendizagem colaborativa nas aulas de laboratório.

No entanto, a maioria dos alunos (80\%) apresentou percepções boas/muito boas sobre os assuntos abordados nas aulas teóricas destinadas à melhor compreensão/articulação com as práticas (Figura 3B). Nesse caso, os dados não mostraram diferença entre experimentos presenciais e remotos.

Para prosseguir com o modo remoto, muitos aspectos tiveram que ser adaptados pelos professores do curso de TransQuim, considerando-se todas as especificidades. A Figura 4 mostra as percepções dos alunos sobre a qualidade dos materiais disponíveis para acompanhar o curso na modalidade remota, bem como a disponibilidade do professor e ainda a forma de avaliação utilizada durante este período. 
Figura 4. Percepção dos alunos sobre a qualidade dos materiais do curso do laboratório disponibilizados no modo remoto, a disponibilidade do professor e o método de avaliação no período remoto.

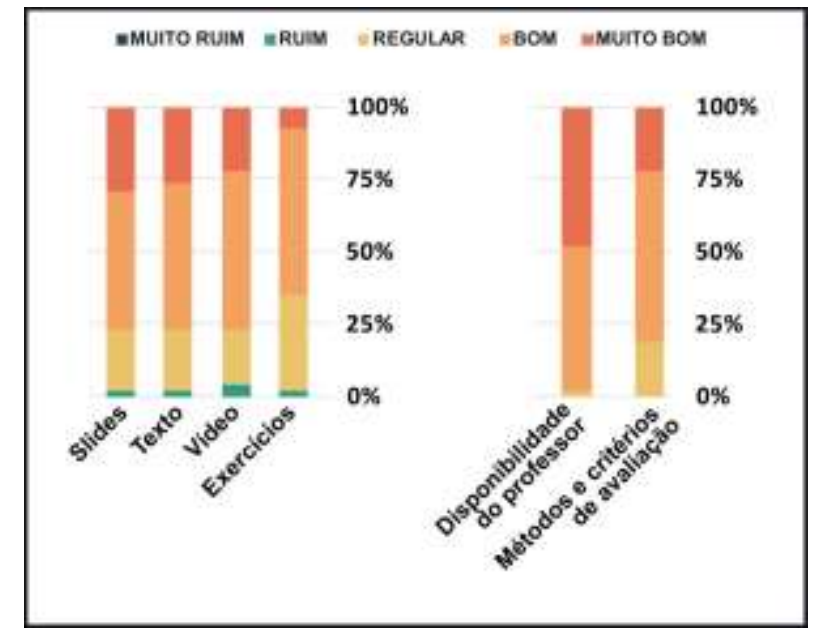

Fonte: Autores (2021).

Esses dados sugerem que os alunos apreciaram a nova estrutura do curso, destacando a boa qualidade dos materiais. Quase todos os alunos (97\%) sinalizaram bom/muito bom para a disponibilidade de seus professores, mostrando que os professores estavam atentos aos problemas de comunicação e estavam dispostos a responder rapidamente a qualquer dúvida em reuniões síncronas ou por e-mail, tal como relatado por outros autores (Asrar et al., 2018; Zan, 2019). Vale ressaltar que as aulas teóricas foram realizadas em modo síncrono. Assim, os professores estavam disponíveis online, podendo responder prontamente às dúvidas de seus alunos, sob demanda, facilitando o processo de aprendizagem. Yu (2021) desenvolveu uma abordagem interessante usando dados coletados durante o período pandêmico, identificando também que a personalidade dos alunos pode afetar o seu processo de aprendizagem e superar os comportamentos tímidos e introvertidos, acrescentando que a interação síncrona com tais alunos poderia minimizar essas dificuldades.

Quando os alunos foram questionados sobre o método de avaliação aplicado no curso ofertado no modo remoto, a maioria (80\%) avaliou como bom/muito bom. A mudança mais significativa no tipo de avaliação nesta modalidade, em comparação com a modalidade presencial, foi que os alunos tiveram que trabalhar individualmente para elaborar os relatórios de laboratório. As percepções favoráveis da avaliação dos alunos significam que os objetivos primários do curso foram razoavelmente alcançados.

Alguns comentários espontâneos dos alunos sobre a disponibilidade de seus professores puderam ser notados:

"[...] a disponibilidade para esclarecer dúvidas por e-mail me ajudou no processo”;

"Entender as aulas de laboratório no modo ECE foi mais complicado, mas também acredito que o trabalho realizado foi muito bom, o material fornecido foi de boa qualidade. O problema era a visualização dos experimentos.”

Devido à mudança do modo presencial para remoto, foi necessário compreender as facilidades e dificuldades expressas neste contexto. Este tópico será abordado a seguir.

\subsection{Impressão dos estudantes sobre a mudança do curso presencial para o remoto: facilidades e dificuldades}

Os comentários dos alunos foram analisados, considerando-se as aulas teóricas e de laboratório separadamente. Quanto às aulas teóricas, os alunos destacaram alguns aspectos da mudança das aulas presenciais para as remotas. De maneira geral, foram feitos comentários positivos, como pode ser acompanhado a partir dos relatos a seguir: 
"[...] o professor trabalhou muito para ministrar uma boa aula; também foi engraçado por causa de algumas atividades dinâmicas que realizamos durante a aula";

"O curso de TransQuim apresentou uma mudança muito suave; meu professor utilizou excelentes métodos de ensino e avaliação”.

No entanto, alguns alunos apontaram insatisfação com as aulas no modo remoto:

"[...] muitas aulas se tornaram mais monótonas e exaustivas do que seriam pessoalmente”;

"As aulas não tiveram o mesmo impacto (quando comparadas com o presencial), mas foram as melhores possíveis para mim, dentro do contexto".

Quando os alunos foram questionados sobre suas dificuldades em acompanhar a mudança das aulas teóricas presenciais para as remotas, alguns pontos foram relatados, tais como:

"[...] conciliar os horários das aulas com a rotina doméstica, dificuldade de compreensão da resolução dos exercícios e falta de motivação para assistir às aulas [...]”;

"(falta de) interação com os professores";

"[...] entender como funciona a plataforma escolhida e como enviar atividades por meio dela”.

Uma questão relacionada à adaptabilidade também foi comentada nos seguintes relatos dos alunos:

"Minha casa nunca tinha sido um lugar de estudo individual, com muitas distrações, e isso acabou sendo uma dificuldade";

“(Dificuldade de) Adaptação para estudar e acompanhar aulas em casa”.

As facilidades apontadas pelos alunos durante a mudança foram: o horário flexível para realização das atividades; acesso fácil a uma aula gravada e possibilidades de revisão de vídeos sempre que quiserem; procurar outras fontes a qualquer momento; tempo economizado no deslocamento para chegar à universidade e sem estresse de mobilidade. Também foi comentado que "o acesso a material dos nossos professores ficou mais fácil e útil para esclarecer algumas questões".

Em relação às aulas de laboratório, os alunos relataram como principal dificuldade na mudança das aulas presenciais para as remotas a impossibilidade de realização do experimento prático, expondo uma decepção particular, como pode ser observado nos relatos dos alunos:

"Não foi possível interagir com os materiais do laboratório (reagentes, vidrarias e equipamentos) e dúvidas sobre o procedimento experimental não foram esclarecidas";

"Dificuldades de compreensão de experimentos";

"Os experimentos não eram realmente práticos".

Os alunos também relataram dificuldades em compreender os próprios experimentos. Um exemplo é explicitado a seguir:

"Embora houvesse muitas explicações, às vezes não era muito claro o motivo pelo qual estávamos fazendo isso, mas logo após uma explicação adicional, o experimento poderia ser realizado sem problemas”.

Algumas outras questões, como aprendizado sobre o conteúdo, comprometimento com as tarefas e relatórios de laboratório com muitas questões incertas foram levantadas pelos alunos. 
Os aspectos positivos da alteração para as aulas de laboratório remoto relatados foram: a facilidade para fazer relatórios de laboratório, ter mais tempo para se dedicar aos cursos e a possibilidade de consultar outras fontes, além dos próprios colegas, como pode ser verificado nas seguintes afirmações:

"Os relatórios de laboratório foram essenciais para fazê-lo e cobrir todo o conteúdo";

"[...] mais tempo disponível para se dedicar ao curso, já que no ensino remoto não há problemas de mobilidade, o que demoraria muito mais nas aulas presenciais";

"Facilidade para consultar algum outro conteúdo online durante a aula";

"Poder consultar mais colegas e tirar dúvidas em comum, além de poder se ajudar melhor".

No entanto, vários alunos foram enfáticos ao afirmar que não tiveram nenhuma facilidade no modo remoto apenas porque "o modo presencial é muito melhor", o que concordamos plenamente.

\subsection{Percepção dos alunos sobre o aprendizado durante as aulas de laboratório}

Tanto as experiências de laboratório presenciais quanto em atividades remotas implicaram em diferenças quanto às percepções do processo de aprendizagem dos alunos. Considerando as escalas positivas, a maioria dos alunos ( 90\%) apontou um desempenho bom/muito bom quanto aos laboratórios presenciais, contra cerca de metade dos alunos (55\%) com a perspectiva remota (Figura 5).

Figura 5. Autoavaliação dos alunos em relação à aprendizagem em cada prática. Experiências 1 a 3: oferecidas no modo presencial; Experiências 4 a 6: oferecidas no modo remoto.

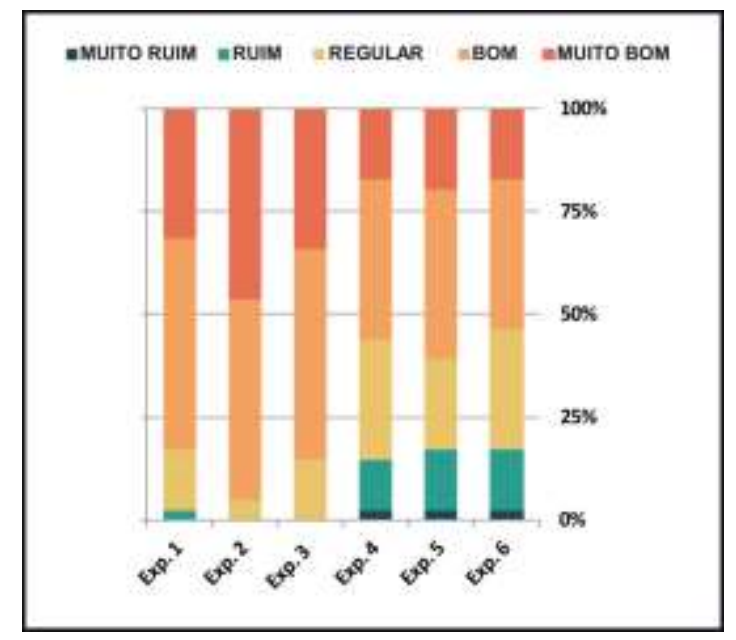

Fonte: Autores (2021).

Esse resultado reforça a importância da modalidade presencial, suportadas pelas interações entre professor-alunos e entre os próprios alunos durante seu processo de aprendizagem, conforme demonstrado por outros autores (Jefffery \& Bauer, 2020; Wart et al., 2020).

Da mesma forma, com base nas avaliações do relatório, cerca de 75\% dos alunos apresentaram melhor desempenho nos laboratórios presenciais do que nos ofertados de forma remota. Esta diminuição no desempenho dos alunos, diagnosticada por meio da autoavaliação (Figura 5) e notas dos relatórios, pode ser explicada pelo trabalho mais individual do aluno, fato que requer certa autonomia e comportamento emocional durante o ensino remoto na modalidade ECE. Por outro lado, esse comportamento também pode indicar que o aprendizado colaborativo durante os laboratórios presenciais é mais evidente do que o aprendizado individual estimulado durante laboratório remoto, tal como reportado por Davenport et al. (2018). 
Porém, alguns alunos relataram perda de estímulos e desafios para ajudá-los a aprender os conceitos e realizar tarefas de modo pleno, como pode ser percebido no seguinte relato:

"(A condução da disciplina no modo remoto) foi bom, mas, muitas vezes, o objetivo principal era cumprir as tarefas de aquisição de conceitos, e isso não é propriamente aprender."

Quando questionados se problemas de espaço comprometeram o desempenho no curso, metade dos alunos relatou que sim, tais como os explicitados a seguir:

"Eu moro com muitas pessoas. Apesar do espaço adequado que tenho para estudar, muitas pessoas por perto e a responsabilidade pelas tarefas domésticas dificultam o foco";

"A falta de um espaço adequado para estudar certamente afetou meu desempenho. Além da instabilidade da internet para tarefas totalmente online".

No entanto, algumas respostas destacaram o esforço despendido como positivo, como pode ser observado no seguinte relato: "A busca de uma melhoria na acessibilidade também me ajudou a impulsionar meu desempenho no curso."

Embora os conteúdos abordados nos experimentos tenham sido diferentes entre os modos presencial e remoto, eles estavam relacionados a diferentes conceitos abordados na disciplina. Assim, a percepção dos alunos sobre a importância das aulas de laboratório para compreender os conceitos foi abordada tanto no modo presencial (Figura 6A, Experimentos 1 a 3 ) quanto no modo remoto (Figura 6B, Experimentos 4 a 6).

Figura 6. Autoavaliação dos estudantes sobre como os conceitos químicos estudados na teoria foram melhor compreendidos nas seções de laboratório: A) Presencial (Experimentos 1 a 3) e B) Remoto (Experimentos 4 a 6).

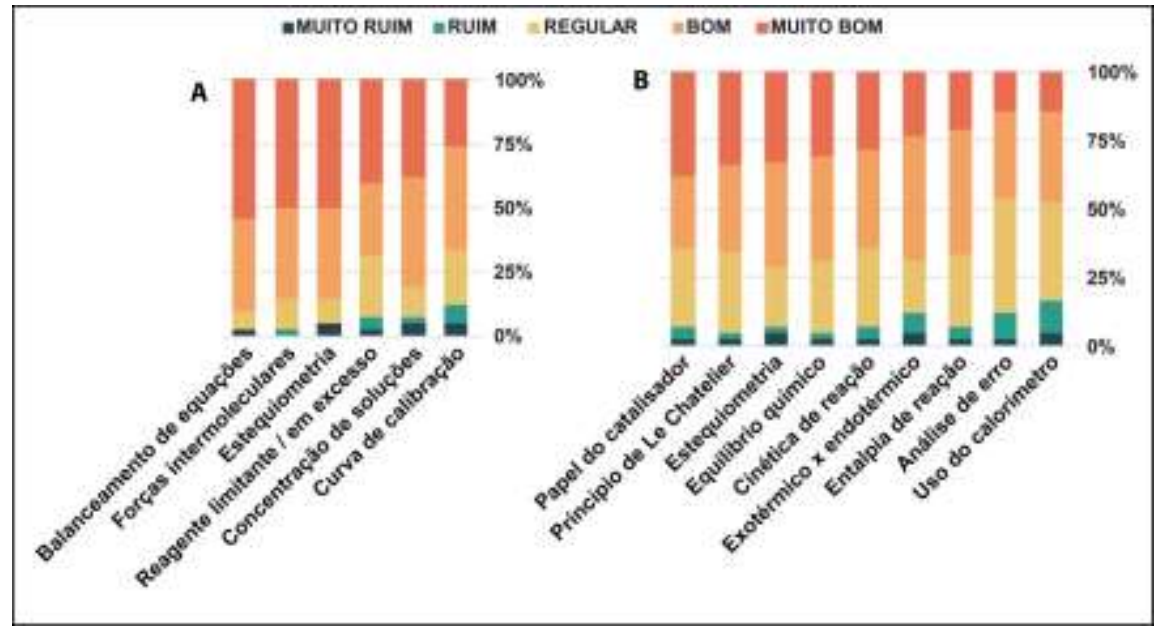

Fonte: Autores (2021).

Considerando o modo presencial (Figura 6A), cerca de $80 \%$ dos alunos relataram uma percepção positiva quanto à melhor compreensão dos conceitos teóricos após as aulas de laboratório. Essa percepção foi evidenciada no experimento que envolveu balanceamento de equações químicas (90\%).

Por outro lado, a partir das aulas remotas (Figura 6B), um pouco mais da metade dos alunos (60\%) conseguiu entender os conceitos da teoria após os laboratórios remotos, considerando a mesma escala perceptiva. Essa diferença pode ser atribuída a vários motivos, com destaque para: (i) as experiências adquiridas durante os laboratórios presenciais terem sido 
diferentes das remotas devido ao contexto do ambiente prático; (ii) em um laboratório remoto, os professores abordaram alguns conceitos que não eram profundamente explorados na teoria. Por exemplo, o item análise de erro, em que menos da metade dos alunos (45\%) declarou compreender as relações entre tais tópicos por meio de uma abordagem teórico-prática. O experimento referia-se à determinação da entalpia da reação de decomposição do peróxido de hidrogênio, em que era necessário traçar um gráfico de temperatura versus tempo, determinando a variação máxima de temperatura, para cálculo da entalpia de reação e comparação do resultado calculado com o disponível na literatura. A análise crítica do desvio entre esses valores poderia ser explicada por ajustes experimentais, como adaptação de um calorímetro construído em laboratório ou erro de leitura de temperatura. Esses tópicos estão diretamente relacionados à experiência prática desenvolvida em laboratório, o que dificultou a compreensão dos alunos; (iii) laboratórios remotos realizados individualmente. Nestes casos, o desempenho depende fortemente dos procedimentos experimentais e do estabelecimento de inter-relações entre conceitos teóricos. No entanto, a importância do laboratório remoto pode ser verificada analisando o conceito de estequiometria de reação, presente nos dois modos de ensino (gráficos da Figura 6 A e B): 85\% e 70\% dos alunos declararam que compreenderam bem este conceito, respectivamente para os modos presencial e remoto, fato que se refere às limitações do curso.

\subsection{Percepção dos alunos sobre a autoavaliação.}

Quando foram questionados sobre seu interesse em cada uma das experiências (Figura 7), foi possível notar uma ligeira queda no interesse em experimentos de laboratório remoto (4 a 6) quando comparados aos experimentos presenciais (1 a 3), revelado pelo aumento das percepções negativas.

Figura 7. Avaliação do interesse dos alunos em cada experimento. Experiências 1 a 3, oferecidas no modo presencial; Experiências 4 a 6, oferecidas no modo remoto.

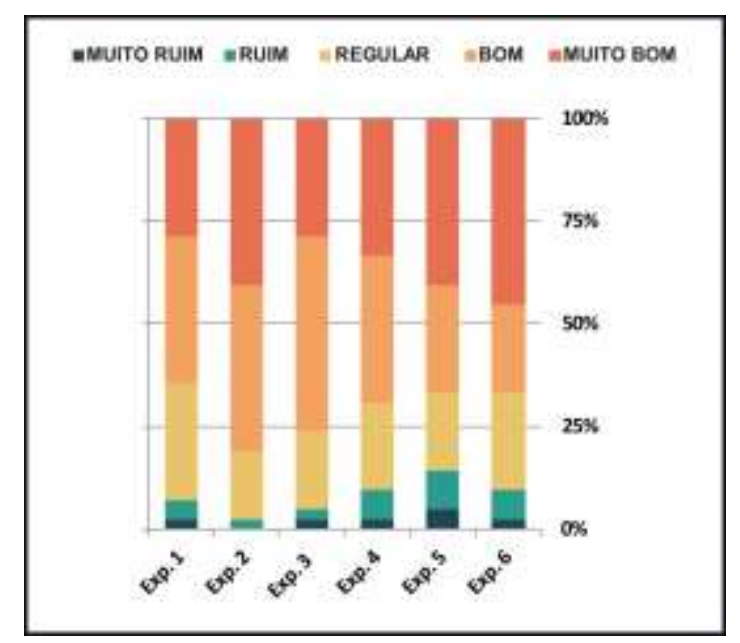

Fonte: Autores (2021).

Por outro lado, a possibilidade de visualizar experimentos remotos usando materiais interessantes chamou a atenção dos alunos. Eles se autoavaliaram com grande interesse em práticas remotas, evidenciado por suas percepções positivas. Entre os laboratórios remotos com excelente percepção, o experimento Le Chatelier (6) despertou mais interesse, uma vez que os materiais disponíveis eram provavelmente mais atraentes e estimulantes. Neste caso, vídeos online sobre mudança de cor a partir de reações de formação complexas foram fornecidos. Quanto aos experimentos presenciais, as interações intermoleculares (2) ganharam destaque. Foi possível estabelecer uma relação direta entre as interações intermoleculares com as propriedades físicas dos materiais, como mudanças de estado físico das substâncias, ponto de ebulição e tensão superficial. 
A partir da autoavaliação dos alunos durante o laboratório de ECE (Figura 8), pode-se verificar que eles não apresentaram dificuldades quanto às habilidades cognitivas, como leitura de artigos científicos, interpretação gráfica, compreensão experimental e análise de dados.

Figura 8. Autoavaliação do aluno sobre o desenvolvimento de habilidades cognitivas (A), atitude / postura (B) e gerência das atividades (C) durante o curso de laboratório no ECE.

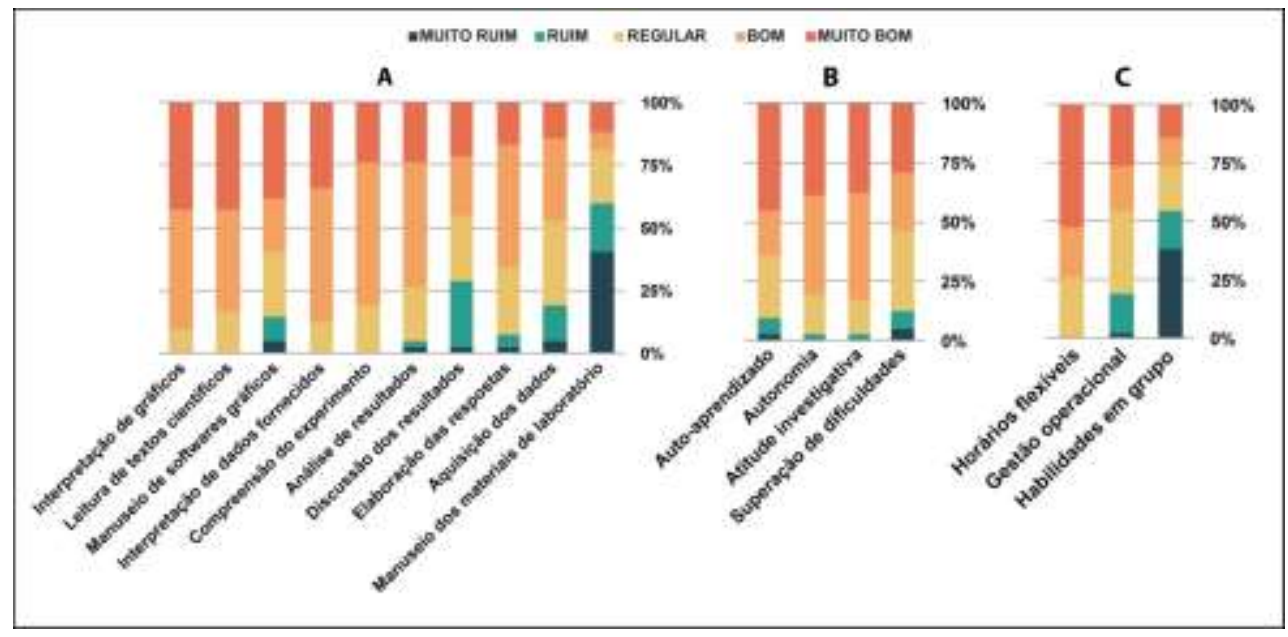

Fonte: Autores (2021).

Algumas habilidades que dependem do laboratório parecem ter sido prejudicadas considerando-se as percepções discentes, como exemplo, podemos citar o manuseio de equipamentos de laboratório e discussão dos resultados (Figura 8A), apontados com escalas negativas por $\sim 60 \%$ e $\sim 30 \%$ dos alunos, respectivamente. Também apontaram algumas outras questões como, por exemplo, dificuldades no manuseio de softwares gráficos, análise dos resultados, elaboração das respostas e aquisição de dados. Por outro lado, alguns itens como interpretação de gráficos, leitura de textos científicos, interpretação de dados fornecidos e a compreensão dos experimentos foram bem avaliados na percepção dos discentes, não havendo indicação de ruim/muito ruim, como pode ser observado na Figura 8A.

Em contrapartida, quanto aos itens atitude e postura, os discentes reconheceram tanto autonomia quanto atitude investigativa (80\%) como pontos favoráveis durante o curso de laboratório nesta modalidade, porém sinalizando alguma adversidade por terem que ser autodidatas, no item autoaprendizado (65\%) e também quanto à superação de dificuldades ( 50\%) (Figura 8B).

Como as atividades foram realizadas individualmente, as habilidades em grupo e a gestão operacional também parecem ter sido prejudicadas pelo modo remoto (Figura 8C) nas questões de laboratório. No entanto, horários flexíveis foram indicados como um fator positivo ( 75\%), como também observado em outros grupos de pesquisa (Lee et al., 2017; Wart et al., 2020). Entretanto, pela percepção dos discentes, um item que parece ter sido bastante prejudicado foi o de habilidades em grupo, com aproximadamente a metade dos estudantes sinalizando esta dificuldade durante as aulas de laboratório no modo remoto.

Vale ressaltar que os alunos desta universidade, em geral, apresentam problemas de mobilidade urbana, gastando horas no deslocamento de casa para a universidade. Nesse cenário, as aulas remotas possibilitaram um melhor gerenciamento do tempo. 


\subsection{Percepção dos estudantes quanto ao curso em geral}

Comparando as percepç̃es sobre dedicação e desempenho, houve maior dedicação aos laboratórios presenciais do que aos experimentos oferecidos na forma remota (Figura 9A).

Figura 9. Percepções dos alunos sobre A) dedicação e desempenho: comparação entre o modo presencial e remoto; B) visão geral do curso.

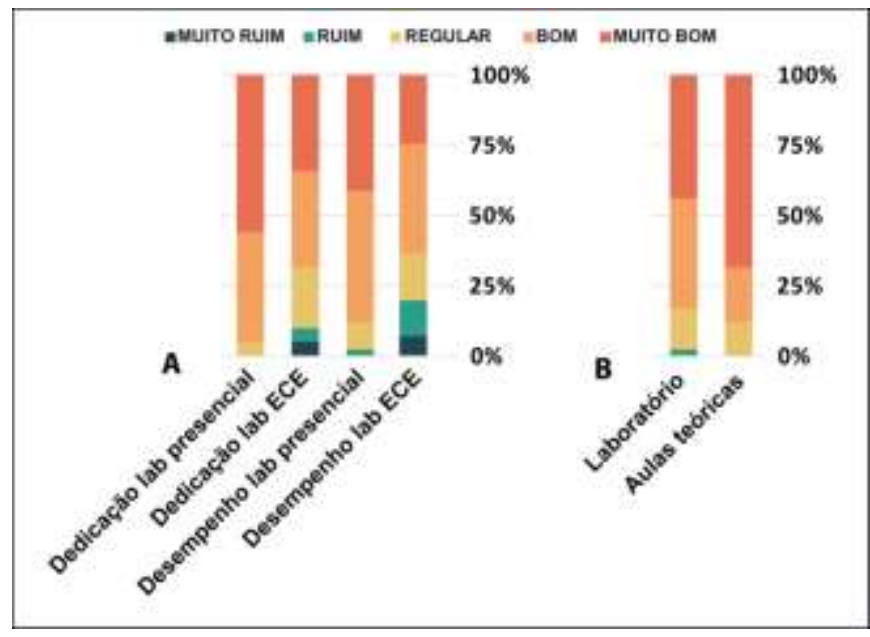

Fonte: Autores (2021).

Esse comportamento pode ser consequência da ausência do laboratório físico, combinando componentes afetivos com um ambiente de aprendizagem que pode desencorajar a estudar de forma remota.

Quanto às percepções dos alunos sobre a visão geral do curso, a maioria declarou impressões positivas considerando teoria e laboratório (Figura 9B), como pode ser verificado nos seguintes relatos:

"O modo ECE foi bem executado. TransQuim foi um dos melhores cursos ofertados, mas, de qualquer forma, poderia ser melhorado";

"Excepcional! Parabéns aos professores!"

Ainda de acordo com a percepção de nossos alunos, mesmo tendo uma avaliação menos favorável associada ao modo remoto, quando comparado ao modo presencial, os resultados gerais mostraram-se positivos (Figura 9B). Os alunos também tiveram a oportunidade de se expressar com comentários espontâneos confirmando:

(i) a importância do laboratório:

"Infelizmente, as atividades práticas foram muito prejudicadas. Presencialmente, eram muito melhores. As atividades teóricas, porém, permaneceram no mesmo nível, com boas aulas";

"A aula prática a distância não consegue suprir todo o intuito que uma aula prática presencial faria".

"[...] mas no geral eram piores do que nas aulas presenciais, principalmente quanto às aulas práticas”.

(ii) a estrutura da ECE: "O curso foi bem estruturado, apesar das dificuldades"

(iii) as lacunas de comunicação inerentes ao processo: "Acredito que feedbacks mais frequentes sobre as atividades de laboratório seriam benéficos para a compreensão dos alunos [...]” 
Por fim, percebe-se a importância deste trabalho, já que tocamos profundamente nossos alunos:

"Gostei da introdução ao manuseio de vidrarias usadas no laboratório... gostei da parte teórica, mesmo na modalidade ECE. Aprendi muito, mais do que esperava, e me interessei mais por química".

\section{Considerações Finais}

TransQuim é a primeira disciplina de química com laboratório oferecido pelo curso do Bacharelado em Ciência e Tecnologia da Universidade Federal do $\mathrm{ABC}$, em que os alunos se sentem motivados e envolvidos com o ambiente investigativo proposto durante essas aulas de laboratório. No cenário de adaptação da ECE, foi possível analisar as percepções sobre o curso como um estudo de caso. Avaliamos os impactos de ministrar um curso experimental, originalmente planejado para acontecer presencialmente, e repentinamente adaptado ao modo remoto.

Os principais desafios enfrentados pelos professores foram lidar com as novas tecnologias de comunicação, adequando seus materiais ao modo remoto, além de manter o engajamento dos alunos. Os alunos também realizaram modificações em seus espaços e rotinas de forma a garantir sua participação nessas aulas remotas. Ao longo do período de adaptação, perceberam a manutenção de aulas teóricas como adequadas. No entanto, a perda foi significativa nos laboratórios, relacionada à ausência de manipulação de equipamentos de laboratório, desenvolvimento de habilidades em grupo e experiências de aprendizagem colaborativa. Apesar de permanecerem interessados, os alunos perderam as oportunidades de realização das aulas em um ambiente de aprendizagem mais adequado. No entanto, esses desafios parecem ter sido superados com sucesso com base em uma avaliação geral positiva sobre o curso. Relataram o desejo e a comodidade de concluir o curso, mesmo à distância, para não atrasar a carreira e começar a trabalhar imediatamente. Em geral, os resultados apresentados demonstraram as percepções dos alunos semelhantes aquelas já relatadas por outros grupos ao redor do mundo.

Vivenciamos um período difícil, em que foi observado um processo de vacinação lento da população, que incluiu professores, alunos e funcionários. Salas e laboratórios cheios provavelmente ainda serão desencorajados por um certo período. Assim, no atual cenário de pandemia, o curso de TransQuim pôde ser oferecido inteiramente no modo remoto. Sendo este o caso, entender as percepções de nossos alunos sobre como as mudanças do curso presencial para o modo remoto afetaram seu aprendizado, interesse e desempenho é essencial para o planejamento deste novo curso. Suas dificuldades e adaptações para o aprendizado devem ser consideradas. Uma alternativa a médio prazo pode ser a oferta de tal curso no modelo híbrido, porém considerando-se todas as limitações envolvidas. Assim, parte das aulas teóricas poderiam ser oferecidas à distância e os laboratórios poderiam ser realizados de forma mista, com alguns experimentos planejados presencialmente e outros mantidos de forma virtual. Em geral, os professores devem encontrar alternativas de uso de plataformas virtuais para melhorar o relacionamento e a comunicação com seus alunos, bem como entre eles, para manter o engajamento, o interesse e a motivação. Ao mesmo tempo, utilizando metodologias de aprendizagem mais ativas, os alunos seriam solicitados a se tornarem mais protagonistas de seu processo de aprendizagem, o que significa um fator positivo para o seu sucesso.

Algumas observações devem ser consideradas neste trabalho, como exposto na sequência:

1) O curso de TransQuim inclui vários professores e alunos distribuídos em muitas turmas. Este questionário foi realizado com apenas uma pequena parte do grupo ativo, por dois professores de laboratório, que certamente apresentam diferenças quanto às estratégias de ensino;

2) O preenchimento do questionário foi voluntário e opcional, justificando a baixa adesão; a pouca aderência não permite uma análise estatística. Os alunos que não responderam ao questionário podem ter um perfil diferente em relação à nossa amostra. 
3) Nem todos que cursaram a disciplina de TransQuim têm interesse em seguir a carreira de química, o que pode explicar as dificuldades apontadas no questionário;

4) Os alunos deveriam ter feito as experiências remotas individualmente, mas não podemos garantir este cenário.

5) As aulas de laboratório remoto podem limitar o estímulo para um comportamento investigativo aos que costumam assumir uma atitude mais passiva durante as aulas, levando à perda do senso crítico e argumentativo.

Esperamos poder contribuir para estimular outras comunidades que ainda estão longe de assumir a nova normalidade a moldar seus cursos, proporcionando aos seus alunos a oportunidade de continuar seus estudos remotamente, almejando assim um bom emprego no futuro próximo. Recomenda-se a realização de mais estudos semelhantes, a fim de melhor compreender os impactos da pandemia nos cursos de graduação, principalmente aqueles que contêm atividades laboratoriais.

\section{Agradecimentos}

Os autores agradecem aos alunos da disciplina de Transformações Químicas que responderam ao questionário, colaborando com este estudo. Agradecemos também à nossa colega Profa. Dra. Patrícia Dantoni, coordenadora do curso. As instalações da UFABC são muito apreciadas, principalmente os laboratórios didáticos, técnicos e monitores, que deram suporte às atividades presenciais. Agradecemos o apoio financeiro da FAPESP (2017/18007-2, 2016/16512-9), agência brasileira de fomento à pesquisa do Estado de São Paulo.

\section{Referências}

Asrar, Z., Tariq, N., \& Rashid, H. (2018). The Impact of Communication Between Teachers and Students: A Case Study of the Faculty of Management Sciences, University of Karachi, Pakistan. European Scientific Journal, 14(16), 32-39. https://doi.org/10.19044/esj.2018.v14n16p32.

Babinčáková, M., \& Bernard, P. (2020). Online Experimentation during COVID-19 Secondary School Closures: Teaching Methods and Student Perceptions. Journal of Chemical Education, 97(9), 3295-3300. https://doi.org/10.1021/acs.jchemed.0c00748.

Blizak, D., Blizak, S., Bouchenak, O., \& Yahiaoui, K. (2020). Students’ Perceptions Regarding the Abrupt Transition to Online Learning During the COVID19 Pandemic: Case of Faculty of Chemistry and Hydrocarbons at the University of Boumerdes - Algeria. Journal of Chemical Education, 97(9), 2466-2471. https://doi.org/10.1021/acs.jchemed.0c00668.

Brasil (2021). Ministério da Educação. Coronavírus. https://www.gov.br/mec/pt-br/coronavirus.

Brasil (2021a). Ministério da Educação. Ações do MEC em resposta à pandemia de Covid-19. http://portal.mec.gov.br/index.php?option=com_docm an\&view=dow nload\&alias=183641-ebook\&category_slug=2020\&Itemid=30192.

Coronavirus (2021). World Health Organization. https://www.who.int/health-topics/coronavirus\#tab=tab_1.

Croda, J., de Oliveira, W. K., Frutuoso, R. L., Mandetta, L. H., Baia-da-Silva, D. C., Brito-Sousa, J. D., Monteiro, W. M., \& Lacerda, M. V. G. (2020). COVID-19 in Brazil: advantages of a socialized unified health system and preparation to contain cases. Journal of the Brazilian Society of Tropical Medicine. https://doi.org/10.1590/0037-8682-0167-2020.

Davenport, J. L., Rafferty, A. N., \& Yaron, D. J. (2018). Whether and How Authentic Contexts Using a Virtual Chemistry Lab Support Learning. Journal of Chemical Education, 95(8), 1250-1259. https://doi.org/10.1021/acs.jchemed.8b00048.

Dickson-Karn, N. M. (2020). Student Feedback on Distance Learning in the Quantitative Chemical Analysis Laboratory. Journal of Chemical Education, 97(9), 2955-2959. https://doi.org/10.1021/acs.jchemed.0c00578.

Dietrich, N., Kentheswaran, K., Ahmadi, A., Teychené, J., Bessière, Y., \& Alfenore, S. (2020). Attempts, Successes, and Failures of Distance Learning in the Time of COVID-19. Journal of Chemical Education, 97(9), 2448-2457. https://doi.org/10.1021/acs.jchemed.0c00717.

Emenike, M. E., Schick, C. P., Van Duzor, A. G., Sabella, M. S., Hendrickson, S. M., \& Langdon, L. S. (2020). Leveraging Undergraduate Learning Assistants to Engage Students during Remote Instruction: Strategies and Lessons Learned from Four Institutions. Journal of Chemical Education, 97(9), 2502-2511. https://doi.org/10.1021/acs.jchemed.0c00779.

George-Williams, S., Motion, A., Pullen, R., Rutledge, P. J., Schmid, S., \& Wilkinson, S. (2020). Chemistry in the Time of COVID-19: Reflections on a Very Unusual Semester. Journal of Chemical Education, 97(9), 2928-2934. https://doi.org/10.1021/acs.jchemed.0c00796.

Guerra Junior, A. L.; Simm, J. F. S.; Pinho, E. de C. S.; \& Nantes, E. A. S. (2021). A eficácia do roteiro de autoestudo como recurso didático no ensino remoto. Research, Society and Development, 10(13), e160101320891. https://rsdjournal.org/index.php/rsd/article/view/20891. 
Hensen, C., Glinowiecka-Cox, G., \& Barbera, J. (2020). Assessing Differences between Three Virtual General Chemistry Experiments and Similar Hands-On Experiments. Journal of Chemical Education, 97(3), 616-625. https://doi.org/10.1021/acs.jchemed.9b00748.

Hodges, C., Moore, S., Lockee, B., Trust, T., \& Bond, A. (2020). The Difference Between Emergency Remote Teaching and Online Learning, EDUCAUSE. https://er.educause.edu/articles/2020/3/the-difference-between-emergency-remote-teaching-and-online-learning.

Howitz, W. J., Thane, T. A., Frey, T. L., Wang, X. S., Gonzales, J. C., Tretbar, C. A., Seith, D. D., Saluga, S. J., Lam, S., Nguyen, M. M., Tieu, P., Link, R. D., \& Edwards, K. D. (2020). Online in No Time: Design and Implementation of a Remote Learning First Quarter General Chemistry Laboratory and Second Quarter Organic Chemistry Laboratory. Journal of Chemical Education, 97(9), 2624-2634. https://doi.org/10.1021/acs.jchemed.0c00895.

Huang, J. (2020). Successes and Challenges: Online Teaching and Learning of Chemistry in Higher Education in China in the Time of COVID-19. Journal of Chemical Education, 97(9), 2810-2814. https://doi.org/10.1021/acs.jchemed.0c00671.

Jeffery, K. A., \& Bauer, C. F. (2020). Students' Responses to Emergency Remote Online Teaching Reveal Critical Factors for All Teaching. Journal of Chemical Education, 97(9), 2472-2485. https://doi.org/10.1021/acs.jchemed.0c00736.

Koirala, D., Silwal, M., Gurung, S., Bhattarai, M., \& Vikash Kumar, K.C. (2020). Perception towards Online Classes during COVID-19 among Nursing Students of a Medical College of Kaski District, Nepal. Journal of Biomedical Research \& Environmental Sciences, 1(6), 249-255. https://dx.doi.org/10.37871/jbres1151.

Kolack, K., Hemraj-Benny, T., \& Chauhan, M. (2020). Community College Chemistry Instruction and Research in the Time of COVID-19. Journal of Chemical Education, 97(9), 2889-2894. https://doi.org/10.1021/acs.jchemed.0c00700.

Kolil, V. K., Muthupalani, S., \& Achuthan, K. (2020). Virtual experimental platforms in chemistry laboratory education and its impact on experimental selfefficacy. International Journal of Educational Technology in Higher Education, 17, \#30. https://doi.org/10.1186/s41239-020-00204-3.

Lee, Y., Stringer, D., \& Du, J. (2017). What determines students' preference of online to F2F class? Business Education Innovation Journal, 9(2), 97-102. http://www.beijournal.com/images/12_V9N2_final-2.pdf.

McCarthy, N. (2020). COVID-19's staggering impact on global education. World Economic Forum. https://www.weforum.org/agenda/2020/03/infographiccovid19-coronavirus-impact-global-education-health-schools.

Ranga, J. S. (2020). Online Engagement of Commuter Students in a General Chemistry Course During COVID-19. Journal of Chemical Education, 97(9), 2866-2870. https://doi.org/10.1021/acs.jchemed.0c00633.

Rodríguez-Rodríguez, E., Sánchez-Paniagua, M., Sanz-Landaluze, J., \& Moreno-Guzmán, M. (2020). Analytical Chemistry Teaching Adaptation in the COVID-19 Period: Experiences and Students' Opinion. Journal of Chemical Education, 97(9), 2556-2564. https://doi.org/10.1021/acs.jchemed.0c00923.

Selco, J. I. (2020). Using Hands-On Chemistry Experiments While Teaching Online. Journal of Chemical Education, 97(9), 2617-2623. https://doi.org/10.1021/acs.jchemed.0c00424.

Soares, R., de Mello, M. C. S., da Silva, C. M., Machado, W., \& Arbilla, G. (2020). Online Chemistry Education Challenges for Rio de Janeiro Students during the COVID-19 Pandemic. Journal of Chemical Education, 97(9), 3396-3399. https://doi.org/10.1021/acs.jchemed.0c00775.

Stufflebeam, D. L., \& Zhang, G. (2017). The CIPP Evaluation Model: How to Evaluate for Improvement and Accountability. Guilford Publications: New York.

Talanquer, V., Bucat, R., Tasker, R., \& Mahaffy, P. G. (2020). Lessons from a Pandemic: Educating for Complexity, Change, Uncertainty, Vulnerability, and Resilience. Journal of Chemical Education, 97(9), 2696-2700. https://doi.org/10.1021/acs.jchemed.0c00627.

Tam, G. \& El-Azar, D. (2020). World Economic Forum. 3 ways the coronavirus pandemic could reshape education. https://www.weforum.org/agenda/2020/03/3-ways-coronavirus-is-reshaping-education-and-what-changes-might-be-here-to-stay.

Tan, H. R., Chng, W. H., Chonardo, C., Tao Tao Ng, M., \& Fung, F. M. (2020). How Chemists Achieve Active Learning Online During the COVID-19 Pandemic: Using the Community of Inquiry (CoI) Framework to Support Remote Teaching. Journal of Chemical Education, 97(9), $2512-2518$. https://doi.org/10.1021/acs.jchemed.0c00541.

TQ (2020). Transformações químicas. YouTube. https://www.youtube.com/playlist?list=PLJIM5YEyF3s14JvyLOs_Xg64rUg4_VZTa.

$\begin{array}{llllllll}\text { UFABC (2020). CONSELHO DE ENSINO, PESQUISA E } & \text { EXTENSÃO. } & \text { Resolução } 239 / 2020 .\end{array}$ Dhttps://www.ufabc.edu.br/images/stories/comunicare/boletimdeservico/boletim_servico_ufabc_932.pdf.

Villanueva, O., Behmke, D. A., Morris, J. D., Simmons, R., Anfuso, C., Woodbridge, C. M., \& Guo, Y. (2020). Adapting to the COVID-19 Online Transition: Reflections in a General Chemistry Sequence Taught by Multiple Instructors with Diverse Pedagogies. Journal of Chemical Education, 97(9), 2458-2465. https://doi.org/10.1021/acs.jchemed.0c00752.

Wart, M. V., Ni, A., Medina, P., Canelon, J., Kordrostami, M., Zhang, J., \& Liu, Y. (2020) Integrating students' perspectives about online learning: a hierarchy of factors. International Journal of Educational Technology in Higher Education, 17, \#53. https://doi.org/10.1186/s41239-020-00229-8.

Wenzel, T. (2020). Collaborative Group Learning in Remotely Taught Analytical Chemistry Courses. Journal of Chemical Education, 97(9), 2715-2718. https://doi.org/10.1021/acs.jchemed.0c00520.

Winkelmann, K., Keeney-Kennicutt, W., Fowler, D., \& Macik, M. (2017). Development, Implementation, and Assessment of General Chemistry Lab Experiments Performed in the Virtual World of Second Life. Journal of Chemical Education, 94(7), 849-858. https://doi.org/10.1021/acs.jchemed.6b00733.

Yu, Z. (2021). The effects of gender, educational level, and personality on online learning outcomes during the COVID-19 pandemic. International Journal of Educational Technology in Higher Education, 18, \#14. https://doi.org/10.1186/s41239-021-00252-3. 
Research, Society and Development, v. 10, n. 17, e15101724184, 2021

(CC BY 4.0) | ISSN 2525-3409 | DOI: http://dx.doi.org/10.33448/rsd-v10i17.24184

Zan, N. (2019). Communication Channel Between Teachers and Students in Chemistry Education: WhatsApp. US-China Education Review A, 9(1), 18-30. http://dx.doi.org/10.17265/2161-623X/2019.01.002.

Zômpero, A. F. \& Laburú, C. E. (2011). Atividades investigativas no ensino de ciências: aspectos históricos e diferentes abordagens. Ensaio: pesquisa em educação em ciências, 13 (3), 67-80. https://doi.org/10.1590/1983-21172011130305. 